\title{
AUTONOMÍA Y UNIDAD EN EL CONOCIMIENTO JURÍDICO*
}

Guillermo Lariguet**

Universidad de Córdoba

\begin{abstract}
"Es pues indispensable que lleguemos a convencernos de que todas las ciencias están tan íntimamente relacionadas, que más fácil es aprenderlas todas a la vez que aprehender una sola, separándola por completo de las demás”. Descartes, René: Reglas para la Dirección del Espiritu, Versión original (1701), Porrúa, México, 2000, p. 96.
\end{abstract}

\section{Introducción}

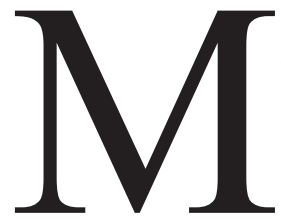

propósito central en este trabajo consiste en indagar el uso que los juristas hacen de la idea de autonomía en la elección de sistemas normativos aplicables y los conflictos posibles que esta idea tiene con la idea de unidad. Aunque ambas ideas, autonomía y unidad, son usadas por los juristas con múltiples y diversos significados, la distinción básica que tendré en cuenta será la siguiente: autonomía y unidad conceptual (para el ámbito del conocimiento jurídico) y autonomía y unidad normativa (para el ámbito del objeto del conocimiento jurídico). La autonomía referida al conocimiento jurídico presupone la existencia de conceptos relacionados

* Este trabajo es producto de mi tesis doctoral (aún no concluida) "Problemas de Autonomía y Unidad en el Conocimiento Jurídico" (bajo la dirección del Profesor Ricardo Caracciolo). Muchos problemas que aquí no serán tratados se encuentran en ella; por ejemplo, la reconstrucción de las nociones de disciplina, interdisciplina, interteoría, la vinculación de estas nociones con el problema del objeto, método y aparatos conceptuales de las dogmáticas, los distintos usos de las nociones de autonomía y unidad, la relación de estas nociones con los procesos de constitucionalización del derecho, codificación y descodificación, etc., A su vez, este trabajo configura un extracto de un trabajo mayor "Acerca de la Noción de Interdisciplina en el Conocimiento Jurídico" (inédito). Este extracto fue expuesto en el XI Seminario Internacional de Teoría y Filosofía del Derecho de Vaquerías (2002).

** Conicet. Centro de Investigaciones Jurídicas y Sociales. U. N. Córdoba, Argentina. Deseo agradecer las detalladas observaciones que los Profesores Ricardo Caracciolo, Pablo Navarro, Jorge Rodríguez, Paolo Comanducci, Jorge Cerdio Herrán hicieron a los borradores previos de este trabajo. A ellos deben añadirse las observaciones puntuales que los Profesores Eugenio Bulygin, Ricardo Guibourg y Ricardo Guarinoni me formularon con motivo de su exposición en Vaquerías. Los errores que pudiesen persistir en el trabajo son de mi entera responsabilidad. 
sistemáticamente ${ }^{1}$ (teorías jurídicas); mientras que la autonomía referida al objeto del conocimiento jurídico presupone la existencia de normas relacionadas sistemáticamente (sistemas normativos). Por supuesto: el carácter sistemático en ambos casos se encuentra "implícito" y, por ello, su explicitación depende de una reconstrucción conceptual determinada ${ }^{2}$.

Las ideas de autonomía y unidad conceptual son versiones de las nociones de autonomía disciplinar e interdisciplina o, con mayor rigor, de las nociones de teoría y relaciones interteóricas. La idea es que los conflictos posibles que estas dos ideas mantienen en el ámbito del conocimiento jurídico están fuertemente relacionados con los conflictos que se plantean en el ámbito del objeto de ese conocimiento: los sistemas normativos.

Estos conflictos pueden originarse en la existencia de situaciones factuales que sean descriptas de manera diversa (sea que estas descripciones formen parte de las teorías dogmático-jurídicas, sea que estas descripciones formen parte del antecedente (o supuesto de hecho) de las normas jurídicas. En ambos casos, las descripciones son o no son distintas porque el empleo de los conceptos con que caracterizo esas situaciones factuales repercute en la identificación conceptual de casos idénticos o distintos.

El problema es que si a partir de las situaciones factuales incluidas en el antecedente de las normas reconstruyo casos idénticos, podría generarse un problema difícil: que un mismo caso sea solucionado con normas que lleven a resultados diferentes. De aquí surge la relevancia de los conceptos construidos por los juristas. Esta relevancia se muestra diáfanamente en que del hecho de que un mismo término (por ejemplo, el término 'ambiente') se exprese con el mismo lenguaje en sistemas normativos diferentes (por ejemplo en el derecho civil, penal y ambiental) ello no impide que este término pueda ser reconstruido conceptualmente en casos idénticos o distintos que conduzcan a procesos judiciales que, según el concepto del que se parta, lleven a resultados idénticos o distintos.

Para desarrollar el propósito central de este trabajo, la estructura expositiva será la siguiente. En primer lugar, mostraré sucintamente de qué manera los juristas usan sus teorías al momento de delimitar conjuntos de casos y normas admisibles para resolverlos. La idea es que el uso de las teorías (sea

${ }^{1}$ La parte conceptual de una teoría se complementa con una parte descriptiva que presupone una relación sistemática entre proposiciones. Para esta cuestión puede verse Aarnio, Aulis: "On Changes in the Systematics of Law", Rechstheorie, 11, 1986, pp. 161-170; Alchourrón, Carlos: "Systematization and Change in the Science of Law", Rechststheorie 11, 1986, pp. 171-184 ; Frändberg, Ake: "An Essay on the Systematics of Legal Concepts", Scandinavian Studies in Law, Vol. 31, Almqvist \& Wiksell International, Stockholm, 1987, pp. 83-115.

${ }^{2}$ Véase Guibourg, Ricardo: "El Papel de las Teorías en la Cultura Jurídica de Nuestro Tiempo", en Pensar en las Normas, Eudeba, Bs As, 1999, p. 171. 
en forma descriptiva o sea en forma práctica) dota de racionalidad a esta instancia y por ello debería ser posible encontrar maneras de controlar racionalmente proposiciones descriptivas o prescriptivas del tipo "en el caso C es aplicable la Norma N" o "en el Caso C 'debe' ser aplicable la Norma N". La cuestión del control racional es un problema abierto todavía en la ciencia jurídica y en la epistemología en general. No me ocuparé de él aquí directamente, pero sí de dos aspectos con los que se encuentra relacionado. El análisis de estos dos aspectos constituye un paso previo a la discusión del problema del control racional de estas proposiciones. El primer aspecto estará referido a discutir las relaciones posibles entre sistemas normativos y sistemas teóricos y, por lo tanto, discutir las relaciones entre los componentes "descriptivos" y "prácticos" del conocimiento jurídico. Estas relaciones no son pacíficas y explican en parte la dificultad de analizar el problema del control racional de las proposiciones arriba mencionadas. Para esta discusión tomaré un ejemplo del derecho tributario y lo examinaré desde tres alternativas metateóricas distintas. Aunque en la discusión del primer aspecto se encuentra presupuesto un análisis de los posibles conflictos que las ideas de autonomía y unidad mantienen entre sí con relación a los sistemas normativos que deben considerarse aplicables, el análisis explícito de estos conflictos integrará el segundo aspecto que me propongo considerar. Estos conflictos son expresiones de desacuerdos ${ }^{3}$ entre los juristas; desacuerdos que parecen explicar el supuesto carácter conflictivo de la ciencia jurídica ${ }^{4}$. El análisis de los desacuerdos es importante para la discusión del

\footnotetext{
${ }^{3}$ Estoy pensando en los de índole semántica y axiológica. Ambos desacuerdos conspiran contra la posibilidad de lograr unificaciones mayores en los ámbitos del conocimiento jurídico y su objeto. Con respecto a los primeros, un ejemplo ilustrativo lo suministra Sucar con respecto a la 'teoría del dolo' en la dogmática civilista argentina, donde el término 'dolo' se vincula con tres significados diferentes que son producto de tres teorías jurídicas diferentes, las teorías del "cumplimiento de las obligaciones", "hechos ilícitos" y "vicios de la voluntad". Cfr. Sucar, Germán: "Niveles de Análisis en la Teoría del Derecho", Inédito. Los desacuerdos axiológicos, surgidos en hipótesis de lagunas axiológicas o de contradicciones pragmáticas, se relacionan con normas reconstruidas como "principios jurídicos", donde lo que se debate es qué principio permite reconstruir al "derecho" bajo su mejor aspecto.

${ }^{4}$ Carácter "conflictivo" que parece constituir un rasgo acusado de la "ciencia del derecho'" qua ciencia social. De esta manera, la situación de la ciencia del derecho tiene un "parecido de familia" con la situación que Geertz describe para otra ciencia social como la antropología caracterizada más por "el refinamiento de los desacuerdos que por el perfeccionamiento de los acuerdos". Geertz, Clifford: "Thik Description: Toward an Interpretative Theory of Culture", en Readings in the Philosophy of Social Sciences, Edited by Michael Martin and Lee C. McIntyre, A Bradford Book, MIT Press, 1994, p. 230. El ejemplo de Geertz al caracterizar la antropología resulta muy ilustrativo. Después de todo, los antropólogos y los juristas comparten -y discuten- problemas similares, formulados en preguntas como las siguientes: ¿cuál es la manera más “fiable” de 'explicar' o ‘describir' una práctica social? ¿Observando? ¿Participando? Este mismo tipo de preguntas enfrenta a los juristas; enfrentamientos que son explicitados a
} 
control racional porque ellos parecen presuponer la existencia de múltiples y diversos criterios sobre normas y sistemas normativos aplicables. La existencia de esta multiplicidad de criterios suele generar dos reconstrucciones filosófico-jurídicas que se encuentran conectadas con un debate epistemológico general. Según la primera, debemos ser modestos y considerar que la racionalidad de una proposición es relativa al criterio escogido por el jurista (relativismo epistemológico ${ }^{5}$ ). Según la segunda, debe ser posible reconstruir una doctrina unificada de estos criterios de aplicabilidad ${ }^{6} \mathrm{y}$ encontrar una racionalidad común a todas las proposiciones (racionalismo epistemológico).

Por lo ya expuesto no me demoraré en estas cuestiones y, como es obvio, no examinaré todos los tipos de desacuerdos que los juristas mantienen. Solo me concentraré en un desacuerdo relacionado con una laguna normativa. A tal efecto analizaré un caso de embargo y trataré de mostrar cómo de la defensa de la autonomía o su negación depende la posibilidad o imposibilidad de reducciones intersistemáticas ${ }^{7}$ en los dos niveles descriptos anteriormente (objeto de conocimiento y conocimiento).

\section{EI uso de las teorías jurídicas para delimitar casos y normas}

Los juristas emplean sus teorías con múltiples funciones. Una de ellas consiste en usarlas para precisar el antecedente de la norma (que describe cierto hecho que puede representarse por una variable, por ejemplo, p) a partir del cual es posible construir casos caracterizados por ciertas propiedades. La interpretación de $\mathrm{p}$ es relevante porque repercute en la determinación de las circunstancias fácticas del problema normativo. En tal sentido resulta

través de los debates metateóricos o filosóficos como los que mantienen Hart y Dworkin. Para Hart, los juristas practican satisfactoriamente su labor teórica cuando se constriñen a describir la práctica de los participantes; para Dworkin, cuando se enderezan a justificar bajo la mejor teoría político-moral posible, la práctica de los participantes. Los sociólogos (Giddens, Anthony y Turner, Jonathan. H: "Introducción”, en La Teoría Social Hoy, Alianza, Madrid, 1995, pp. 9-21), los historiadores (White, Hayden: Metahistoria, FCE, Bs As, 1998, pp.11;31), etc., mantienen desacuerdos prácticamente idénticos. Tras las discusiones sobre cómo delimitar su objeto de conocimiento, cómo determinar una base empírica o cómo explicar mejor un fenómeno, suelen encontrarse desacuerdos de esta índole, especialmente dos tipos de desacuerdos que en la práctica forman un continuo: desacuerdos epistémico normativos (cómo debería ser el mejor modelo explicativo) y desacuerdos político-normativos (por ejemplo, cómo debería ser "modificada" la sociedad).

${ }^{5}$ Morey, Patricia: "Racionalismo y Relativismo de la Explicación. Hacia el Pluralismo", MS, Inédito.

${ }^{6}$ Véase Navarro, Pablo; Orunesu, Claudina; Rodríguez, Jorge y Sucar Germán: “La Aplicabilidad de las Normas Jurídicas", Analisi e Diritto, 2000, p. 152.

7 Véase Nickles, Thomas: "Two Concepts of Intertheoretic Reduction", The Journal of Philosophy, Vol. LXX, Nº 7, 1973, p. 184. 
admisible pensar, como Navarro y Rodríguez, que "la calificación normativa de una acción (por ejemplo, $\mathrm{Oq}$ ) es sensible a la variación del contexto fáctico en que se trama el problema normativo". Es decir, "el status normativo de una acción puede variar cuando se modifica el conjunto de casos considerados, aun cuando las normas del sistema permanezcan constantes"8.

Como intentaré mostrar cuando discuta un problema de embargo, el cambio de niveles de casos está relacionado directamente con la capacidad de los sistemas normativos de resolver en forma completa o coherente los problemas normativos.

En otras palabras, la interpretación de p como una "condición de aplicación" suficiente o no suficiente es relevante para determinar si el status deóntico de una acción determinada (por ejemplo, q) es derrotado o se mantiene. Y la interpretación de p como condicional de aplicación derrotable o no derrotable depende intrínsecamente del modo en que los juristas usen sus teorías al momento de reconstruir el problema normativo, su contexto fáctico y las normas aplicables ${ }^{9}$. Entonces, la tarea dogmática de interpretación de p se realiza con la ayuda de conceptos y teorías jurídicas. Esta tarea es importante y puede volverse sofisticada ${ }^{10}$ cuando el antecedente de la norma contiene conceptos abiertos o potencialmente abiertos. Esta tarea es relevante para identificar las normas con las que se construirán los microsistemas que resuelvan los problemas así como las combinaciones de normas que se consideren admisibles para responder al problema normativo ${ }^{11}$. Esto es así porque el producto de la asignación de significados a estos componentes puede ser útil para suministrar criterios de elección de las normas internamente aplicables al problema normativo; estos criterios, en principio, son los significados de estos componentes. Digo "en principio" porque

\footnotetext{
${ }^{8}$ Navarro, Pablo y Rodríguez, Jorge: "Derrotabilidad y Sistematización de Normas Jurídicas", Isonomía, No. ${ }^{\circ} 13$, México, 2000, p.65.

${ }^{9} \mathrm{~A}$ los fines del presente trabajo presupongo la tesis normal según la cual una norma $\mathrm{N}$ es aplicable si N pertenece al Derecho x y es internamente aplicable. No es mi pretensión discutir en este contexto los problemas que surgen para esta tesis.

${ }^{10}$ Que consiste en utilizar las teorías para reconstruir conjuntos de casos posibles y normas aplicables. Conforme esta idea las teorías jurídicas tienen una función "mediadora" que se interpone entre el contenido genérico de las normas y los casos posibles a los que se aplican las normas. Esta idea está arraigada en la dogmática fuertemente. Véase, por ejemplo, Bacigalupo, Enrique: Principios de Derecho Penal, Akal ediciones, Madrid, 1994, p. 12.

${ }^{11}$ De manera que los juristas emplean sus teorías no sólo para sistematizar sino para identificar las normas que integrarán esos sistemas. Es esto lo que lleva a algunos filósofos a sostener que una adecuada reconstrucción filosófica del empleo del "método jurídico de conocimiento" comprende no sólo la sistematización sino la identificación de las normas que se sistematizarán. Cfr. Aguiló Regla, Josep: Teoría General de las Fuentes del Derecho (y del orden jurídico), Ariel, Barcelona, 2000, p. 147.
} 
para determinar las normas aplicables los juristas pueden usar sus teorías no para elucidar el significado de los componentes de la norma sino para determinar la fuerza justificatoria (o 'force') de las normas ${ }^{12}$. Gran parte de los desacuerdos que los juristas mantienen en el interior de una disciplina dogmática determinada o entre disciplinas dogmáticas diversas se basa, para seguir el vocabulario de Dworkin, en la existencia de concepciones divergentes acerca de estos dos aspectos ${ }^{13}$.

\section{Las relaciones entre sistemas normativos y sistemas teóricos}

Como sugerí en las secciones anteriores, los sistemas normativos están fuertemente correlacionados con los sistemas teóricos pues las teorías colaboran en la determinación de los casos y sistemas normativos admisibles para ellos. En ocasiones, estos últimos son imperfectos debido a la existencia de defectos semánticos ${ }^{14}$, lógicos ${ }^{15} \mathrm{o}$ axiológicos ${ }^{16}$. Tratándose de sistemas normativos que se aplican al mundo, la existencia de estos defectos es una posibilidad inerradicable. El carácter imperfecto o potencialmente imperfecto de los sistemas normativos hace difícil saber cuándo los juristas usan sus teorías de manera descriptiva o práctica.

En otras palabras, es difícil saber dónde termina lo teórico y dónde comienza lo práctico. Para entender cómo ante estas imperfecciones los juristas usan sus teorías pensemos en un caso en que el fisco procure gravar la deuda de una sociedad x. Supongamos que la legislación tributaria contiene un concepto de pasivo supuestamente aplicable a este caso particular; concepto que constituye el cimiento en que el fisco fundamenta su pretensión. La sociedad puede creer que este cimiento no es sólido. La razón de esta creencia se basa en el hecho de que la legislación tributaria no tiene un concepto muy preciso de pasivo y en este caso existen dudas acerca de si este concepto tributario de pasivo se aplica o no a este caso. Supongamos que las mismas normas del sistema tributario admiten la posibilidad de una

\footnotetext{
12 Para una revisión "interna" del positivismo a partir de esta propiedad enfatizada por Dworkin, puede verse Waluchow, Will: Inclusive Legal Positivism, Oxford University Press, Oxford, 1994, 42-46.

${ }^{13}$ Dworkin, Ronald: Law's Empire, Harvard University Press, Cambridge, Mass, 1986, pp. 70-72.

${ }^{14}$ Por ejemplo, lo que Alchourrón y Bulygin denominan "lagunas de reconocimiento". Alchourrón, Carlos y Bulygin, Eugenio: Introducción a la Metodología de las Ciencias Jurídicas y Sociales, Astrea, Bs As, 1987, pp. 61-65.

${ }^{15}$ Por ejemplo, lo que Alchourrón y Bulygin ( op.cit., pp. 41-49) denominan "lagunas normativas".

${ }^{16}$ Por ejemplo, lo que Alchourrón y Bulygin (op.cit., pp. 156-167) denominan "lagunas axiológicas" (o "ideológicas" o "políticas" en la jerga de los juristas).
} 
búsqueda de conceptos de pasivo en otros sectores normativos. Supongamos que el derecho civil tenga un concepto más preciso y que una reconstrucción dogmática conduzca a negar autonomía conceptual al derecho tributario $\mathrm{y}$, en consecuencia, permita reducirlo conceptualmente a un sistema de derecho civil.

En el caso examinado, la existencia de una laguna de reconocimiento en el sistema tributario habilitó la negación de autonomía de éste y la reducción de este sistema a un sistema de derecho civil con un concepto de pasivo preciso. La existencia de una laguna de reconocimiento en un sistema habilitaría la sustitución de éste por otro sistema que carezca de este defecto. Los juristas tributaristas podrían haber evitado esta reducción ofreciendo una definición estipulativa de pasivo que incluyera la deuda de la sociedad. Como toda estipulación presupone una proposición analítica que, como tal, expresa una verdad necesaria, este procedimiento, que Alchourrón y Bulygin llaman de "subsunción genérica", habría evitado en forma inobjetable la reducción ${ }^{17}$.

Frente a esto, es posible que la división de las dogmáticas en disciplinas que se consideran "autónomas" obedezca al propósito de delimitar un conjunto de reglas semánticas que impidan que, por simple estipulación, un caso sea subsumido en una norma con consecuencias determinadas (por ejemplo, en una norma del derecho civil que excluya la posibilidad de gravamen de la deuda). Es probable, también, que la autonomización de disciplinas (y la disputa entre disciplinas dogmáticas) no obedezca pura y exclusivamente a la delimitación de reglas semánticas sino a ciertos propósitos valorativos ${ }^{18}$ que los dogmáticos creen que deben ser perseguidos (por ejemplo que los gravámenes son necesarios para el sostenimiento de la administración del Estado).

En cualquier caso, ambos tipos de autonomía (de reglas semánticas y propósitos valorativos) son los escollos que una empresa filosófica de unificación total $^{19}$ del derecho y del conocimiento del derecho debe enfrentar.

${ }^{17}$ Permitiendo la aplicación del sistema tributario. Cfr. Alchourrón, Carlos y Bulygin, Eugenio: "Los Límites de la Lógica y el Razonamiento Jurídico", en Carlos Alchourrón y Eugenio Bulygin, Análisis Lógico y Derecho, Centro de Estudios Constitucionales, Madrid, 1991, pp. 304-309.

${ }^{18}$ La repercusión de estos propósitos se hará más clara cuando en la próxima sección trate el problema del conflicto entre sistemas y la existencia de principios axiológicos que, de acuerdo con estos propósitos, los juristas usan para identificar estos sistemas.

${ }^{19}$ La objeción común contra la utilidad y posibilidad racional de una unificación de esta clase se basa en el dato efectivo según el cual los juristas trabajan sobre problemas normativos limitados que no exigen una empresa de unificación total sino la construcción de sistemas parciales para resolver esos problemas. Con independencia de esta objeción creo que esta empresa 
Pero dejemos estas cuestiones a un lado y formulemos la siguiente pregunta: cuando los juristas autonomizan un conjunto de casos y normas aplicables ¿a qué ámbito refieren esta autonomización? ¿A las normas? ¿A los conceptos? La pregunta intenta sugerir la existencia de una correlación estrecha entre sistemas normativos y teóricos en la práctica efectiva de conocimiento $^{20}$. Esta correlación podría expresarse del siguiente modo: "la autonomización de normas presupone el uso de conceptos dogmáticos, pero para autonomizar estos conceptos el jurista parte de las normas que debe autonomizar".

Este razonamiento podría hacernos pensar que el ideal de completitud, en este caso semántico, se aplica a dos dominios que en la práctica están fuertemente relacionados: el del conocimiento del derecho y el del objeto de conocimiento. En este sentido, aunque es analíticamente posible encontrar distinciones entre ambos sistemas, pareciera que son siempre los sistemas normativos (y no los sistemas teóricos) los que resultan prioritarios para el examen de coherencia o completitud. Pero aunque fuese correcto afirmar que esta prioridad y estrecha relación son síntomas de la naturaleza genuina del derecho entendido como el resultado de una práctica compleja construida conjuntamente por legisladores, dogmáticos y jueces, no sería verosímil pensar que la existencia de un defecto en un sistema normativo, por ejemplo una laguna de reconocimiento, se extrapola inmediatamente a un sistema teórico correlativo. Así, en el caso de la deuda de la sociedad, puede ser correcto afirmar, a la vez, que el sistema normativo tributario no contenía una norma que regulase de manera precisa este caso pero que la dogmática tributarista disponía de una teoría del concepto de pasivo. ¿Pero es esta 'teoría' descriptiva de una norma? Conceder a esta pregunta una respuesta afirmativa resulta problemático porque existen dudas acerca de si tal norma del sistema normativo tributario es o no aplicable; dudas que condicionan la aplicación del principio de bivalencia. Sobre la base de las normas de este sistema no es posible decidir si la norma es o no aplicable. Entonces, ¿qué sentido tiene afirmar que la teoría del concepto de pasivo es descriptiva de una norma?

tiene algunos sentidos que, reconstruidos, pueden tornar manejable la empresa. Sin embargo, no desarrollaré en este trabajo esa reconstrucción.

${ }^{20}$ Robustecida, entre otras cosas, por lo que sostiene Caracciolo: "la identificación entre ciencia jurídica y objeto de conocimiento se plantea cuando la cuestión de los fundamentos del conocimiento y la idea de sistema que la acompaña se disuelven tradicionalmente en el problema de los fundamentos de las normas, que vendrían a constituir el material de conocimiento jurídico". Cfr. Caracciolo, Ricardo: "Los Fundamentos del Derecho y los Fundamentos del Conocimiento del Derecho", Crítica, México, Vol. XI, Nº.31,1979, pp. 38-39. 
A esta afirmación podría objetársele que, sí es verdad que existe una correlación fuerte entre sistemas normativos y sistemas teóricos, sí es verdad que son los sistemas normativos los que interesan prioritariamente y sí es verdad que el sistema normativo tributario tiene una laguna de reconocimiento, el sistema teórico no puede ser descriptivo de una norma, a menos que contenga una proposición normativa falsa que afirme que tal laguna no existe en el sistema normativo. Bajo esta proposición se encontraría un procedimiento de "modificación" del sistema normativo tributario.

Esta objeción es importante porque permite mostrar la existencia de una concepción que presupone el carácter puramente "reflejo" y "subalterno" de los sistemas teóricos en la "ciencia del derecho". Esto es así porque, de acuerdo con esta objeción, si los sistemas normativos son incompletos, por reflejo, los sistemas teóricos, en cuanto tales, sólo deben constreñirse a registrar estos defectos de los sistemas normativos. Tras esta objeción se encuentra una posición positivista ${ }^{21}$ para la cual las teorías jurídicas son instrumentos observacionales de registro de la existencia de sistemas normativos y defectos relativos a estos sistemas ${ }^{22}$. Esta posición puede ser cuestionada sosteniendo la afirmación fuerte según la cual, ante imperfecciones semánticas, lógicas o axiológicas relativas a sistemas normativos determinados, los juristas no usan sus ‘teorías' en forma observacional sino práctica. Pero aunque se admita que el carácter observacional de las teorías jurídicas constituye un rasgo típico y prominente de ellas, la afirmación fuerte puede ser sustituida por otra más débil. Esta última afirmación puede sostener que las teorías jurídicas intervienen no sólo ante imperfecciones de los sistemas normativos sino en su misma constitución. La idea es que los sistemas normativos constituyen materiales "brutos" 23 que el jurista termina de moldear con sus conceptos. Por consiguiente, los 'sistemas normativos' son productos de tareas interpretativas en las que los juristas rara vez prescinden de sus conceptos o teorías ${ }^{24}$.

Ante los razonamientos anteriores pareciera que la idea de correlación fuerte entre sistemas normativos y teóricos especula con la existencia de dos grandes dificultades ya indicadas en la introducción: por una parte, la

\footnotetext{
${ }^{21}$ Como creo que es la sostenida, por ejemplo, por los Profesores Alchourrón y Bulygin.

${ }^{22}$ Como he tratado de mostrar en "La Reconstrucción de las 'Teorías Dogmático-Jurídicas' como "Cálculos Interpretados". El problema del carácter empírico de la identificación de normas" (trabajo premiado en el 2001 por la Asociación Argentina de Filosofía del Derecho). Será publicado en el Anuario N.2 (2002) de la Asociación.

${ }^{23}$ Tanto más si se admite que este material está compuesto, ab initio, por "formulaciones normativas" y no por normas.

${ }^{24} \mathrm{Y}$ si esto se admite, se vuelve problemático determinar los criterios que permitan distinguir cuándo el jurista conoce neutralmente el derecho y cuándo lo modifica.
} 
dificultad de distinguir en la práctica efectiva de conocimiento entre los componentes "teóricos" y "prácticos", por otra parte, la dificultad de caracterizar en qué consisten estos componentes ${ }^{25}$. Para poder entender mejor estas dificultades, los juristas podrían proponer las siguientes tres alternativas metateóricas.

La primera alternativa podría sostener que el carácter "teórico" de este sistema es dudoso. Esta duda surge del hecho de que no sabemos si la identificación de la "relevancia" del caso de pasivo, dudosamente previsto, es de carácter "descriptivo" o "prescriptivo". Esta duda es destacada por Caracciolo. Aunque él piensa en el problema de las lagunas normativas y mi ejemplo en lagunas de reconocimiento, pienso que sus razonamientos pueden ser esclarecedores para la cuestión que intento discernir. Para Caracciolo el principal problema "consiste en saber si la expresión "relevante" puede ser usada descriptivamente en relación a los casos no previstos". Una respuesta negativa, argumenta Caracciolo, implica, en principio, que no es posible formular un juicio del tipo "el caso $\mathrm{C}$ es una laguna en el sistema $\mathrm{S}$ " sin presuponer un juicio de valor a tenor del cual es posible formular el enunciado prescriptivo "el caso $\mathrm{C}$ debe ser relevante en el sistema $\mathrm{S}$ " que equivaldría, entonces, a "el caso $\mathrm{C}$ debe estar previsto normativamente en S". "A la inversa, si se pudiera utilizar de manera descriptiva el calificativo "relevante" con respecto a casos no previstos, estos dos enunciados prescriptivos no serían equivalentes; el último vendría a tener un mero signifi-

\footnotetext{
${ }^{25}$ Ambas dificultades son las que vuelven problemáticos los diversos intentos de clasificar la dogmática bajo el canon de otras ciencias (empírico-formales, por ejemplo). Bajo este canon se ha distinguido analíticamente entre las proposiciones descriptivas y justificatorias de una teoría jurídica, sosteniendo que sólo las primeras son racionalmente controlables. Aunque no voy a discutir aquí los presupuestos de esta afirmación, pienso que es necesario encontrar y discutir maneras de controlar racionalmente proposiciones justificatorias. Para realizar estas tareas puede ser inadecuado sostener a priori la falta de carácter "teórico" o "científico" de estas proposiciones. En todo caso, prefiero dejar abierta esta cuestión sugiriendo que ella podría ser explorada con herramientas distintas a las ofrecidas tradicionalmente por la filosofía analítica, por ejemplo, con herramientas de la filosofía pragmatista (Véase, por ejemplo, Putnam, Hilary: El Pragmatismo (especialmente el artículo sobre "La Vigencia de William James", Gedisa, Barcelona, 1999; también Razón, Verdad e Historia, (especialmente el Cap. VI), Tecnos, Madrid, 1988). El pragmatismo ha insinuado que existen muchos casos en que los enunciados factuales y evaluativos se compenetran. Esta compenetración (o continuidad) no significa que la intención del pragmatismo sea negar la posibilidad y utilidad de distinguir analíticamente, esto es, por definición, el status de uno u otro enunciados. La idea del pragmatismo es que los enunciados evaluativos no remiten a entidades que hagan imposible encontrar algún tipo de control racional. Esta sugerencia puede explicar porqué pienso que se puede discutir en términos pragmáticos el "dogma" según el cual proposiciones "prescriptivas" de lege o sententia ferenda (como las que subyacen a la teoría de la alternativa tres, no puedan, al menos, contar con algún tipo de control racional).
} 
cado técnico: el caso en cuestión debe estar previsto si se quiere alcanzar el ideal puramente racional de la completitud"26.

Por su parte, la segunda alternativa podría sostener que la teoría es "conceptualmente" cognoscitiva pero en un sentido inocuo para la práctica dado que si mantenemos el status epistémico "descriptivista" de la teoría, el concepto dogmático de pasivo no podría ser usado como la "solución formalizada" de un caso regulado en forma dudosa por las normas. Con esto no quiero decir que del hecho que un sistema normativo contenga una laguna se sigue que una teoría jurídica la contenga. $\mathrm{Si}$ el sistema tributario $\mathrm{S}$ tiene una laguna porque no tiene una norma que regule el pasivo, podría ocurrir que el sistema teórico tributario T sí tenga una respuesta para ese caso, respuesta incluida en su concepto de pasivo. Pero aquí nos enfrentamos a la objeción que presenté con respecto al sentido de la afirmación según la cual, frente a un sistema normativo defectuoso por razones semánticas, lógicas o axiológicas, un sistema teórico contiene "respuestas" que resuelven descriptivamente los problemas normativos para los cuales un sistema normativo contiene respuestas contradictorias o carece de respuestas (prescriptivas) o existen dudas sobre cuáles son esas respuestas. Uno podría sostener que estas respuestas de las teorías jurídicas carecen de rasgo "descriptivo". La teoría es usada para modificar el sistema normativo tributario $\mathrm{S}$ agregando por medio de conceptos una norma que regule esa situación de pasivo. Este agregado no elimina la existencia de laguna; el agregado presupone un procedimiento que integra una laguna previamente descripta y el resultado de esta integración modifica ese sistema, produciendo un nuevo sistema que lo sustituye (donde el defecto desaparece). Pero en el modelo descriptivista, si la respuesta es "teórica", el concepto de pasivo de la teoría no podría ser usado para colmar la laguna, al menos no en nombre de una "ciencia" (según la visión de este modelo descriptivista de ciencia) ${ }^{27}$. En todo caso, la teoría contaría con una parte puramente descriptiva que permitiría afirmar que en el sistema normativo $\mathrm{S}$ existe una laguna y la parte conceptual quedaría inhabilitada en este modelo, sería inocua para la práctica. Si es claro lo que intento expresar, este modelo descriptivista es demasiado restringido y no da cuenta de cosas importantes que los juristas hacen con sus teorías; para esto es posible usar otro modelo (el justificacionista) que presupone otra consideración de las teorías científico- sociales no positivista ${ }^{28}$. Pe-

${ }^{26}$ Caracciolo, Ricardo: "El Concepto de Laguna. Descripción o Prescripción”, en La Noción de Sistema en la Teoría del Derecho, Fontamara, México, 1994, p. 30; Rodríguez, Jorge: "Axiological Gaps and Normative Relevance" (uso la versión no publicada).

${ }^{27}$ Por ejemplo, la visión proyectada por Ross, Alf: Sobre el Derecho y la Justicia, Traducción de Genaro Carrió, Eudeba, Bs As, 1994, p. 306.

${ }^{28}$ En este análisis me parece trivial discutir la cuestión terminológica de si esta concepción debiera abandonar el uso de la expresión 'teoría'. 
ro antes de optar por este modelo podría ser propicio considerar una alternativa adicional para defender el modelo descriptivista, afirmando que tiene perfecto sentido pensar que un sistema teórico tributario sea descriptivo de una norma mientras que el sistema normativo de origen no contenga esa norma o existan dudas sobre qué norma contiene. Como se recordará, este sistema remitía, en caso de laguna, a otros sistemas que contuviesen una definición de pasivo que permitiera identificar una norma para el caso. Siendo así, podría darse la situación en que el dogmático tributarista realizara una reconstrucción teórica que incluyera proposiciones normativas sobre normas de estos otros sistemas $\mathrm{y}$, por este motivo, su teoría fuera descriptiva pese a los defectos del sistema original. Este sistema teórico podría representarse del siguiente modo:

En el derecho tributario existe una laguna de reconocimiento con respecto a si el concepto de pasivo de este sistema puede aplicarse o no a la deuda de una sociedad $x$

En casos de laguna el derecho tributario remite a sistemas de derecho civil

En el derecho civil el pasivo de una sociedad está definido por las propiedades p, q y r,

Existe una norma en el derecho civil que prescribe que los casos de pasivo definidos por estas propiedades deben quedar excluidos de cualquier gravamen fiscal,

La deuda de la sociedad $x$ queda cubierta conceptualmente por las propiedades $\mathbf{p}, \mathbf{q} \mathbf{y} \mathbf{r}$,

\section{La deuda de la sociedad $x$ no puede ser gravada por el fisco}

Dado que el sistema normativo tributario contiene una laguna, el jurista puede construir una teoría (como la arriba ejemplificada) que le permita elegir otro sistema normativo que resuelva el problema. Esto haría inteligible la idea de que los juristas, en repetidas situaciones, deben determinar cuál sistema teórico es el más adecuado en la elección del sistema normativo que resuelva en forma completa o consistente el caso $^{29}$. La elección puede, como ha mostrado Ihering, estar motivada por la necesidad de "reemplazar" un sistema normativo que los juristas considerar "deficiente" por otro que no consideran tal ${ }^{30}$. Pero

${ }^{29}$ Esta afirmación presupone que en el conocimiento jurídico también existe una fuerte correlación entre los criterios para elegir teorías jurídicas y los criterios para elegir sistemas normativos. (Véase lo que sostiene Caracciolo en nota 20).

${ }^{30}$ von Ihering, Rudolf: "Teoría de la Técnica Jurídica", en El Ámbito de lo Jurídico. Lecturas del Pensamiento Jurídico Contemporáneo, Pompeu Casanovas y José Juan Moreso Editores, Crítica, Grupo Grijalbo Mondadori, Barcelona, 1994, p. 93. 
para identificar esta deficiencia y ofrecer criterios para elegir sistemas normativos que la superen es menester, para Ihering, usar las teorías que construyen los dogmáticos. En caso de que se verificasen algunos de estos defectos en un sistema normativo los juristas, con el auxilio de sus teorías, reemplazarían este sistema por otro. La existencia de este reemplazo permitiría entender el sentido de la "aplicabilidad supletoria" de otros sistemas. Esta aplicabilidad sería una forma interesante de reformular una idea algo oscura de Kelsen según la cual cuando existe una laguna se aplica "todo" el orden jurídico ${ }^{31}$. Tal vez, cuando Kelsen pensaba en esto, su mente distinguía dos cuestiones no siempre advertidas: por una parte, que estas deficiencias son "relativas" a un sistema normativo determinado (son intrasistemáticas), por otra parte, que del hecho que esta deficiencia se verifique en un sistema normativo determinado no significa que no exista otro sistema alternativo que supletoriamente pueda ser aplicado. De ser esta una idea manejable, lo que Kelsen parece querer decir es que el derecho como "orden jurídico" dispone de sistemas con la que resolver de maneras alternativas una misma clase de problemas normativos. Por consiguiente, el acento de Kelsen no se constriñe sólo al carácter intrasistemático de los defectos. La metáfora de aplicabilidad de "todo" el orden jurídico supone que Kelsen examina el problema desde una perspectiva "intersistemática"32. El ejemplo de embargo aeronáutico que suministraré en la próxima sección puede ser muy instructivo a este respecto.

Ahora bien, la tercera alternativa podría sostener que el sistema teórico es conceptualmente cognoscitivo pero no en el sentido descriptivo que acabo de presentar con la segunda alternativa. El sistema es cognoscitivo en sentido prescriptivo ${ }^{33}$, esto es, no en un sentido inocuo para la práctica sino “conceptualmente auxiliar" de la práctica. Sin preocuparse por distinguir normas de proposiciones normativas, este sistema teórico podría contener una proposición prescriptiva que sostuviese que en este caso 'debe' (o debería) aplicarse el sistema normativo $\mathrm{x}^{34}$. Por supuesto, esta tercera alternativa abre un conflicto epistemológico con la segunda. La tercera defiende una concepción "justificacionista” de ciencia según la cual la dogmática no tiene por función "describir" realidad alguna sino justificar qué realidad debe

${ }^{31}$ Alchourrón, Carlos y Bulygin, Eugenio: Introducción a la Metodología de las Ciencias Jurídicas y Sociales, op.cit., p. 187.

${ }^{32}$ Perspectiva que es intuida por los juristas en su uso impreciso de la idea de interdisciplina.

${ }^{33}$ Dado que no es "escéptico" frente a la posibilidad de conocer o controlar racionalmente proposiciones que expresan prescripciones o valores.

${ }^{34} \mathrm{Si}$ en este caso no se concede "local priority" al sistema tributario puede aplicarse otro sistema que garantice una unificación axiológica que presente al derecho "íntegramente" bajo su mejor aspecto. Dworkin, Ronald: Law's Empire, op.cit., pp. 250-254. 
darse. La segunda alternativa defiende una concepción "descriptivista" de ciencia según la cual la dogmática jurídica debe describir en enunciados lógicamente independientes la existencia de enunciados prescriptivos. Para la concepción justificacionista la perspectiva según la cual los sistemas normativos son deficientes en aspectos semánticos, lógicos o axiológicos es producto de la metateoría positivista ${ }^{35}$. Para Dworkin esta metateoría es incompleta pues no da cuenta de la práctica de los juristas. En esta práctica ellos usan sus teorías en forma práctica permitiendo construir sistemas completos y coherentes que presenten al derecho bajo su mejor aspecto. La consideración según la cual los sistemas normativos son incompletos presupone que las teorías jurídicas cumplen una función restringida: registrar estos defectos. El positivismo presupone una concepción epistemológica que, al reconstruir las teorías jurídicas como un repertorio de enunciados empírico-descriptivos "correlativos" de un repertorio de enunciados prescriptivos, no captura la "peculiaridad epistémica" de la labor de los juristas. Esta peculiaridad no consiste necesariamente en negar la existencia de proposiciones normativas descriptivas y el valor que informaciones observacionales fiables tienen en la toma de decisiones prácticas. En realidad, esta concepción sostiene que el rasgo típico y prominente de la ciencia del derecho reposa en su carácter práctico. En esto reside su peculiaridad. Y las concepciones acerca del derecho son o no plausibles si son capaces de dar cuenta la forma en que los juristas usan sus instrumentos conceptuales ${ }^{36}$. Esta tercera alternativa podría mostrar que este uso que los juristas hacen de sus instrumentos conceptuales preserva su carácter teórico, sólo que este carácter es fruto de una concepción no positivista de las ciencias sociales (por ejemplo, la de Habermas ${ }^{37}$ o Dworkin).

\footnotetext{
${ }^{35}$ Para la concepción dworkiniana es precisamente la tarea "interpretativa" del jurista la que permite ver al derecho como un sistema completo " $y$ " coherente. (Cfr. Redondo, Cristina: "Lógica y Concepciones del Derecho", Isonomía, No.13, 2000, p. 42). Es una concepción diferente a la defendida por Alchourrón y Bulygin en Introducción..., op.cit., p. 151. Ellos han mostrado formalmente que la satisfacción simultánea de la completitud y la coherencia es contingente.

${ }^{36} \mathrm{Y}$ si esta concepción metateórica captura los rasgos prominentes de la labor de los juristas podría estar a salvo de las objeciones expresadas por el "argumento del contraste con la práctica" con que ciertos filósofos objetan la plausibilidad de las metateorías positivistas ("duras" o "excluyentes"). Véase Bayón, Juan Carlos: "Derecho, Convencionalismo y Controversia", Inédito.

37 Habermas, Jürgen: Teoría y Praxis. Estudios de filosofia social, Tecnos, Madrid, 1990.
} 


\section{Conflictos entre autonomía y unidad}

En la sección anterior, el análisis de los conflictos entre autonomía y unidad fue indirectamente abordado con relación a una discusión sobre el carácter descriptivo o práctico de las teorías jurídicas. A la inversa, en esta sección, el análisis de los conflictos entre autonomía y unidad será realizado en forma directa, mientras que el análisis del carácter descriptivo o práctico de las teorías jurídicas estará conectado con un análisis más indirecto. Para llevar a cabo el análisis de estos conflictos, supongamos que la situación factual de la que partimos consiste en la interposición de una orden de embargo de un motor de aeronave. Con la ayuda de conceptos dogmáticos yo puedo construir casos diferentes caracterizados a partir de diferentes propiedades. Frente a esta situación podríamos imaginar dos alternativas: una en la que usamos los mismos conceptos y llegamos a identificar las mismas propiedades u otra en que usamos conceptos distintos que nos llevan a identificar distintas propiedades.

En la primera alternativa podríamos llegar a construir el mismo caso. $\mathrm{Si}$ el caso es el mismo para dos sistemas, por ejemplo, el sistema de derecho civil ( $\mathrm{Sc}$ ) y el sistema de derecho aeronáutico $(\mathrm{Sa})$, y estos sistemas tienen normas contradictorias (una en que el embargo está permitido y otra en que el embargo está prohibido), los juristas deberían "ordenar las normas aplicables" dando prioridad a alguna de ellas. La necesidad de dar prioridad a una de las normas y disolver la contradicción conduce a construir sistemas normativos ordenados. Un mismo sistema puede ser ordenado de maneras diversas según el criterio de ordenación que se siga. En este tipo de situaciones, los criterios habituales de ordenación son conocidos bajo las máximas "lex generalis", "lex posterior" y "lex specialis". No es mi cometido discutir el status de estos criterios, sus relaciones conceptuales posibles, ni defender una solución para esta primera alternativa.

De acuerdo con la segunda alternativa podríamos pensar que el uso de conceptos diferentes nos lleva a construir casos caracterizados por propiedades diferentes. Con la propiedad A caracterizo al motor como cosa mueble. En virtud de ello, configuro un nivel de casos uno (C1) de embargo de cosas muebles regulado por normas del sistema civil que permiten (P) el embargo (e), de modo que sea posible afirmar "A $\supset$ Pe"; vamos a suponer que la norma Pe pertenece por el criterio de legalidad al sistema civil ( $\mathrm{Sc}$ ), de modo que sea posible afirmar "C1 $\supset$ Sc". Supongamos que los juristas involucrados con este caso se pregunten qué ocurre si se añaden nuevas propiedades al nivel de casos $\mathrm{C}$, por ejemplo, la propiedad B (donde el motor es caracterizado como una pieza aeronáutica esencial para el funcionamiento de la aeronave). Esta propiedad B podría permitir construir, a partir de la misma situación factual, un nivel de casos dos (C2) de embargo de piezas 
esenciales de aeronaves regulado por normas del sistema aeronáutico que prohiben $(\mathrm{Ph})$ el embargo de aeronaves o piezas fundamentales de éstas (e') por razones tales como "la continuidad del tráfico es de interés público y como tal debe ser asegurada". De manera que "B $\supset$ Phe"”. Suponiendo que la norma Phe' pertenece en virtud del criterio de legalidad al sistema aeronáutico $(\mathrm{Sa})$, sería posible afirmar " $\mathrm{C} 2 \supset \mathrm{Sa}$ ". Ante esta situación yo podría pensar que no existe conflicto alguno pues, sobre la misma situación factual, he construido casos distintos de "embargo"; un caso "genérico" de embargo civil (Ec) caracterizado a partir de la propiedad A y un caso específico de embargo aeronáutico $(\mathrm{Ea})$ caracterizado a partir de la propiedad B; de modo que "Ec $\supset \mathrm{Sc}$ " y si "Ea $\supset \mathrm{Sa}$ ". Ahora bien, aún no nos hemos preguntado si Sa es, por ejemplo, un sistema completo. Supongamos que la situación del motor no estuviese explícitamente regulada por Sa. La primera respuesta que ofrecería la lógica estándar (para la que valen las leyes de refuerzo del antecedente y modus ponens) es que la solución prevista para el nivel de casos uno (C1) constituida por una permisión se mantiene cuando cambio de niveles, por ejemplo, cuando paso al nivel de casos dos. Si el nivel uno estaba caracterizado a partir de la propiedad A y el nivel dos por la propiedad B, podría decir, conforme la lógica estándar regulada por las leyes mencionadas, que si se da la propiedad A aplico la permisión de embargo del Sc; y que, por las leyes de refuerzo del antecedente si "A y B entonces también aplico la permisión del Sc" con lo cual las soluciones del Sa son descartadas ${ }^{38}$. De modo tal que "(A $\left.\left.\supset \mathrm{Pe}\right) \supset \mathrm{A} \cdot \mathrm{B} \supset \mathrm{Pe}\right)$ " ${ }^{\prime 39}$. Confor-

\footnotetext{
${ }^{38}$ Si esto es así, los juristas podrían sentirse francamente perplejos y preguntarse cómo es posible que el Sc que presupone un nivel menos fino que el Sa sea reconstruido como el sistema reductor. Esta perplejidad puede disolverse mediante la comprensión del funcionamiento del teorema de completitud (Cfr. Alchourrón, Carlos y Bulygin, Eugenio: op.cit, pp. 150-151). Según este teorema las soluciones que afectan el universo de casos menos fino se mantienen en el universo de casos más fino.

${ }^{39}$ Esta reconstrucción, a su vez, presupone la existencia de sistemas normativos que se consideran "más generales" que otros. La propiedad A expresa un concepto genérico de embargo civil que es más general que el concepto específico de embargo aeronáutico expresado por la propiedad B. El presupuesto según el cual es aplicable el Sc y no el Sa no es sólo de orden lógico sino epistemológico. En este sentido, un criterio de orden epistemológico para "elegir" sistemas teóricos puede basarse en la mayor capacidad de un sistema en resolver problemas (Cfr. Gómez, Ricardo: "Kuhn y la Racionalidad Científica”. ¿Hacia un kantianismo postdarwiniano?, O. Nudler y G. Klimovsky Compiladores, La Racionalidad en Debate, Vol. I., Centro Editor de América Latina, 1993, p. 150). Pienso que este criterio puede proyectarse sin inconvenientes a los sistemas normativos. Pero, para ello, es preciso advertir la existencia de dos interpretaciones de este criterio que se hallan en pugna. Según una, la mayor capacidad se relaciona con la mayor generalidad de un sistema. Esta interpretación favorece un mayor grado de unidad conceptual en el derecho y su conocimiento. Según la otra, la mayor capacidad se relaciona con la mayor especificidad de un sistema. Esta interpretación favorece la existencia
} 
me con esta reconstrucción B es una propiedad lógicamente "irrelevante", claro está, sólo si el sistema es consistente porque podría ser el caso que la introducción de B estuviese simultáneamente asociada con la permisión y la prohibición, con lo cual B dejaría de ser irrelevante ${ }^{40}$.

Ahora bien, ¿tengo otra manera de reconstruir este conflicto entre el sistema civil y el sistema aeronáutico? Creo que sí. Esta reconstrucción depende de que nos centremos en el uso que los dogmáticos y jueces hacen de la idea de autonomía. En efecto, la idea de autonomía puede ser usada para bloquear la posibilidad de que la solución prevista para el nivel de casos uno, esto es, la permisión del embargo del Sc, se extrapole o se mantenga para el nivel de casos dos caracterizados a partir de la propiedad B. Los juristas pueden usar el argumento de la autonomía para sostener que existen conceptos propios de un sector normativo y razones axiológicas o principios

de sistemas más autónomos. La primera interpretación de este criterio, que es la que subyace a la reconstrucción lógica según la cual es aplicable Sc, permite entender varias cosas. Primero, con relación al derecho, permite dar cuenta de la intuición recurrente de los juristas en torno a la existencia de sectores o principios jurídicos más generales que otros. Segundo, con relación al conocimiento del derecho, permite entender la existencia, en la dogmática misma, de teorías 'generales' del derecho. La mayor generalidad de unas teorías sobre otras sería una manera posible de entender la pretensión de la "teoría civilista del embargo genérico" en 'explicar' mediante una operación de reducción los casos de la "teoría específica del embargo aeronáutico" (Véase Brooks, D. H. M: "How to Perform a Reduction", Philosophy and Phenomenological Research, Vol. LIV, No. 4, 1994, pp. 803-814).

El problema adicional que surge es que el predicado 'explicativo' es ambiguo. Puede referir a la capacidad de una teoría de resolver (descriptivamente) mayor cantidad de casos que otra o puede referir a la capacidad de una teoría de resolver (prescriptivamente) mayor cantidad de casos que la otra. Esto último se hará claro enseguida cuando describa el uso práctico que los juristas hacen de sus teorías a fin de propiciar o impedir la aplicación de determinados sistemas normativos.

${ }^{40}$ Supóngase una norma N1 que correlaciona la solución normativa Pe a los casos identificados por la propiedad A. Si se interpreta esta norma como una norma condicional a cuyo respecto vale el refuerzo del antecedente, entonces cualquier caso A está correlacionado con la solución Pe, con independencia de qué otras propiedades posea. Ahora bien, supóngase que en el sistema también encuentro una norma N2 que correlaciona la solución PHe al caso identificado por la propiedad B. De conformidad con N2, y también por aplicación del refuerzo del antecedente, el caso A y B está correlacionado también con la solución PHe, de manera que ese caso está inconsistentemente normado. A la pregunta de si B es una propiedad relevante respecto de la calificación normativa del embargo, la respuesta es sí. Si esto es así, entonces ¿por qué los juristas suelen asociar la idea del refuerzo del antecedente con la irrelevancia de otras propiedades? Para entender esta asociación habría que manejarse exclusivamente con soluciones maximales (Oe, PHe y Fe) y no minimales como lo es Pe. Supongamos que decimos que un caso está solucionado cuando está correlacionado por el sistema con una solución maximal (de lo contrario habría una laguna parcial). Tomemos como ejemplo para ello un sistema compuesto exclusivamente por la norma N2 que correlaciona la solución PHe al caso B. Si respecto de esa correlación se admite la ley de refuerzo del antecedente, ello no significa que el sistema no admite que se pueda asignar relevancia respecto de la calificación normativa de $\mathrm{B}$ a ninguna otra propiedad, pero sí que no se puede hacerlo SIN GENERAR UNA CONTRADICCION. 
axiológicos ${ }^{41}$ (por ejemplo que la 'aeronave debe cumplir su cometido porque está comprometido un interés público en la continuidad de tráfico') que identifican al Sa de manera distinta al $\mathrm{Sc}^{42}$. De manera que la autonomía del ámbito del conocimiento podría ser usada en dos sentidos que bloquearían el mantenimiento de la solución prevista para el nivel uno de casos en el nivel de casos dos. En un sentido apelando a la existencia de conceptos distintos de embargo que lleven a reconstruir la misma situación factual bajo casos diferentes ${ }^{43}$; en otro sentido apelando a las razones o principios axiológicos que subyacen al caso caracterizado como embargo aeronáutico, en cuyo caso la propiedad B no sería, de acuerdo con el principio mencionado, axiológicamente irrelevante, esto es, su "fuerza" o "peso" sería mayor ${ }^{44}$. En ambos sentidos, la autonomía impide que la solución del Sc se aplique al Sa. Por consiguiente: en el nivel del objeto de conocimiento jurídico, el Sa no puede ser lógicamente reducido al Sc (y lo mismo ocurre respecto de los sistemas teóricos del derecho civil y aeronáutico en el nivel del conocimiento jurídico). La reducción entre sistemas normativos y la reducción paralela entre

${ }^{41}$ Esta autonomía presupone la existencia de dificultades para pensar la unidad conceptual y la unidad práctica no sólo de las dogmáticas entre sí sino de estas unidades en el dominio de cada dogmática.

${ }^{42}$ En muchos casos esta manera de identificar los sistemas presupone un uso "ideológico" de la noción de autonomía tendiente a favorecer la creación o aplicación de normas que responden a intereses particulares de ciertos sectores sociales (Véase Guibourg, Ricardo: “Autonomía", publicado en El Dial, diario jurídico digital, en la semana del 30/3/2000). Esto genera, en los estados de derecho contemporáneos, una tensión entre el ideal de una unidad práctica del derecho y la existencia de intereses fragmentarios. Véase Dworkin, Ronald: op.cit, pp. 179180; 211; 403. Para una discusión de dogmática constitucional puede consultarse Zagrebelsky, Gustavo: El Derecho Dúctil, Trotta, Barcelona, 1999, pp. 13-17.

${ }^{43} \mathrm{Si}$, conforme la autonomía conceptual de los sistemas normativos, los casos son distintos, no es posible la reducción. Esto es importante porque una condición necesaria de la reducción intersistemática es que los casos de los sistemas sean conmensurables.

${ }^{44}$ El Sc también puede ser candidato a la unificación axiológica del derecho, negando, en consecuencia, la autonomía de Sa construida a partir del principio axiológico mencionado. Esto podría ocurrir si se admite que la propiedad A (tratarse de un embargo de cosa mueble sobre la base de un crédito no satisfecho) tiene mayor peso que la propiedad B y, por tanto, tiene mayor "relevancia axiológica", por ejemplo, siguiendo un principio jurídico del derecho civil de mayor alcance que el principio de derecho aeronáutico. Por ejemplo, el siguiente:

De acuerdo con Sc los créditos deben ser satisfechos y "asegurados" por cualquier medio legal

De acuerdo con la definición de Sc $\mathrm{x}$ es un crédito

De acuerdo con Sc el embargo es un medio legal de asegurar la satisfacción de créditos

De acuerdo con Sc el embargo de las cosas muebles para satisfacer créditos está permitido El motor es una cosa mueble

El embargo del motor está permitido 
sistemas teóricos sólo sería posible si negamos la autonomía del sistema aeronáutico. Esto es así porque si la reducción intersistemática configura una herramienta de unificación de sistemas en sistemas más comprehensivos, admitir la reducción es negar la autonomía. Esto se explica porque, por definición, la autonomía pretende delimitar un sistema (normativo o teórico según el caso) que no pueda ser reducido a otro. Una condición necesaria para ello es que este sistema cuente con su propio aparato conceptual (en el que podríamos incluir, por ejemplo, un concepto específico de embargo aeronáutico). Es claro que, como ocurre en el caso que estoy analizando, el Sa puede tener una laguna parcial. Pero de ello, si seguimos la idea de autonomía (y nos independizamos provisoriamente de las conclusiones de la lógica estándar ya descriptas) no se sigue la aplicabilidad del Sc y, por consiguiente, la reducción de Sa a Sc. Podríamos pensar que para que tal reducción sea viable deberían, al menos, ocurrir dos cosas: o bien que Sa no dispusiese de conceptos precisos, sólidos, para describir la situación factual del embargo de motor o bien que Sa para casos dudosos (por ejemplo, en casos de lagunas normativas, de reconocimiento o axiológicas) "remitiese" a la aplicación de sistemas supletorios. Por desgracia ambas situaciones son contingentes. La inexistencia de conceptos sólidos en el Sa no impide que los dogmáticos del derecho aeronáutico dispongan de conceptos precisos para esta situación y los usen para bloquear la solución del Sc. De hecho, el motor de una aeronave está incluido en un concepto de la dogmática aeronáutica según el cual los motores son piezas fundamentales para el funcionamiento de una aeronave. $\mathrm{Y}$ ya sabemos que tanto las piezas de las aeronaves como las aeronaves no pueden ser embargadas en el Sa. Por supuesto que la dogmática podría no tener un concepto preciso. Que las dogmáticas cuenten con aparatos conceptuales desarrollados también es una situación contingente. Pero aún suponiendo que el aparato conceptual de esta dogmática no estuviese plenamente desarrollado, los dogmáticos o los jueces pueden usar las teorías para operar reformulaciones ${ }^{45}$ en el Sa a partir de las cuales obtener principios axiológicos como el ya mencionado: "que las aeronaves deben cumplir su cometido...". Precisamente, frente a lagunas parciales, los juristas interponen sus teorías para obtener estos principios con la finalidad de integrar esas lagunas, impidiendo la aplicación supletoria de otros sistemas. Por su parte, la existencia de una norma de remisión que habilite la aplicación de Sc también es contingente. Aunque ella no exista los juristas pueden usar sus teorías para producir unificaciones axiológicas,

${ }^{45}$ La noción de reformulación, asociada comúnmente con la génesis de "principios generales del derecho", es elucidada por Alchourrón, Carlos y Bulygin, Eugenio: op.cit., pp. 124-133. 
por ejemplo, mediante la reducción de Sa a Sc. Con apoyo en ellas, los juristas podrían argumentar, y de hecho lo hacen, que los principios que identifican Sa deben ceder ante los principios que identifican a Sc todo ello bajo la condición de que los casos de los sistemas sean conmensurables ${ }^{46}$. Por razones estrictamente conceptuales creo que la única condición no contingente de la reducción es esta última: la conmensurabilidad de casos. Si no hay relaciones de implicación entre casos es imposible la reducción. De hecho, la distinción entre niveles de casos menos finos y más finos presupone esta conmensurabilidad ${ }^{47}$.

Ahora bien, la contingencia de estas situaciones revela la necesidad filosófica, por un lado, de dar cuenta de las prácticas efectivas de los juristas $\mathrm{y}$, por otro lado, apreciar que el uso práctico de las teorías jurídicas presupone un conflicto entre autonomía y unidad. Defender sistemas autónomos o defender unificaciones intersistemáticas no depende de cuestiones fijas sino cambiantes.

Sea como fuere y dejando a un lado estas observaciones, creo que es útil aclarar el tipo de autonomía que estoy reconstruyendo. Por lo general, este tipo de autonomía es definida como una autonomía relativa. Un sistema normativo relativamente autónomo puede convivir con lagunas parciales y aún admitir la integración de las lagunas con el "transporte" de normas de otros sistemas (hétero-integración) bajo la concurrencia de dos condiciones necesarias: por un lado, que los conceptos con los que se define una situación factual existan y se apliquen indubitablemente a esta situación, por otro lado, que el sistema remita expresamente a otros sistemas alternativos. La hétero-integración, entonces, presupone una idea de autonomía relativa que es consistente con la noción de completitud (relativa a un universo de casos $\mathrm{y}$ de soluciones de un sistema determinado $)^{48}$.

\footnotetext{
${ }^{46}$ Esto presupone que los juristas suelen efectuar unificaciones empleando sistemas más comprehensivos como el Derecho Civil o Constitucional.

${ }^{47}$ Técnicamente un universo es más fino cuando satisface las siguientes dos condiciones: A) Cuando algún elemento del más fino implica a alguno del menos fino y B) Cuando al menos hay algún elemento del más fino que no está implicado por ningún elemento del menos fino. En este caso el UP2 es más fino que el UP1 porque, además de implicar el elemento A contenido en el UP1, posee un elemento adicional B que no está implicado por ningún elemento del UP1. Véase Alchourrón, Carlos y Bulygin, Eugenio: Introducción..., p.148

${ }^{48}$ Por cierto que es posible encontrar un sentido de autonomía no relativa sino "absoluta"; reconstrucción que en el presente trabajo no desarrollo. Esta autonomía no está relacionada con la idea de completitud (relativa) sino con la idea de "plenitud". Es frecuente la apelación de este sentido en la dogmática penal y, en los siglos XVIII y XIX también lo era en la dogmática civil (Cfr. Alchourrón, Carlos: "On Law and Logic", Ratio Juris, Vol. 9, №.4, 1996, pp. 331-348).
} 
Volviendo al sentido que ocupa el foco de este trabajo, esto es, la autonomía relativa, sostuve que la autonomía podía impedir que las soluciones relativas a un sistema y previstas para un nivel de casos menos fino se mantengan en un nivel de casos más fino. Sugerí que la autonomía nos lleva a una reconstrucción que discrepa con lo que surge de una reconstrucción lógica estándar. Pero, ¿cómo entender la existencia de dos reconstrucciones que llevan a resultados opuestos? ¿Existe un conflicto entre las intuiciones reconstructivas de los filósofos que abrazan la lógica estándar y la intuición de los dogmáticos y jueces que enfrentan estos casos? En principio pareciera que el conflicto existe. Si seguimos la reconstrucción lógica estándar el resultado es la aplicación de la permisión del embargo del derecho civil. Si acatamos las leyes de refuerzo del antecedente y modus ponens y si la propiedad A con que caracterizamos el embargo es adoptada como una condición estricta (o suficiente) la solución prevista para un nivel de casos menos finos (caracterizado por la propiedad A) debe valer para un nivel de casos más finos (en que aparece la propiedad B). La lógica estándar defendida por Alchourrón y Bulygin (que son los filósofos en los que estoy pensando) ha demostrado mediante una serie de teoremas que esto es posible.

Ahora bien, si seguimos la reconstrucción de la dogmática o de los juristas en general, la autonomía impediría que la solución de un nivel menos fino se mantenga en un nivel más fino. ¿Supone esto un rechazo irracional de la lógica por parte de la dogmática? Una primera respuesta podría sostener que no. El conflicto entre ambas reconstrucciones es sólo 'aparente' pues, en rigor, ambas reconstrucciones refieren a "problemas distintos". La reconstrucción lógica estándar centra su poder de explicación conceptual en el problema de la "aplicación de normas" y en suministrar herramientas (alternativas a lógicas basadas en condicionales derrotables) para explicar porqué las soluciones previstas para un nivel menos fino se mantienen incólumes en un nivel más fino. El objeto de esta explicación refiere a la "aplicación de normas". Prueba de ello es que la ley de refuerzo del antecedente se aplica a normas y no sistemas y los problemas de completitud y consistencia son "intrasistemáticos" a tenor de lo cual es posible afirmar, por ejemplo, que el Sa tiene una laguna parcial. Por el contrario, la idea de autonomía seguida por los dogmáticos y jueces centra la cuestión en la "aplicación de sistemas de normas" y en las "relaciones intersistemáticas". Los problemas de la autonomía y unidad remiten a las relaciones entre sistemas y no a las relaciones entre normas. Es cierto que, comprometidos con una lógica estándar monotónica, las soluciones previstas para un nivel menos fino se mantienen en un nivel más fino. Por supuesto que esto es así. Pero la idea de autonomía no apunta a esto. Apunta a las relaciones entre sistemas normativos desarrollando criterios (ciertamente variados y difusos) para mostrar porqué 
es 'racional' aplicar un sistema normativo y no otro o porqué es racional bloquear la aplicación de un sistema dando prioridad a otro. Es claro en este caso que el uso de la expresión 'racional' no se identifica uno a uno con el uso de herramientas de la lógica estándar. En los ejemplos que he examinado los juristas parecen comprometidos no sólo con una racionalidad lógicodeductiva sino con una racionalidad práctica. Esto es evidente en el uso de las teorías tendiente a mostrar el mayor peso o fuerza de los principios de un sector sobre otro. Por supuesto, el compromiso con una racionalidad práctica en principio es independiente de la idoneidad con que los juristas respetan la racionalidad lógica. Digo "en principio" porque la racionalidad práctica podría verse afectada seriamente por un compromiso deficiente con la racionalidad lógica. En efecto, muchos principios que identifican sectores determinados son producto de inferencias inductivas y no deductivas; inferencias realizadas con el fin de cubrir lagunas. El principio según el cual "la aeronave debe cumplir su cometido..." podría ser un ejemplo apropiado de lo que señalo. Ante el carácter "incompleto" de las inferencias inductivas $^{49}$, sería lógicamente viable la aplicación de la permisión del embargo del Sc y no la prohibición del $\mathrm{Sa}$, toda vez que este camino estaría regido por la completitud heredada del universo de casos menos finos al universo de casos más finos. Sin embargo, en la práctica efectiva de los juristas esto no necesariamente ocurre así. Pese al carácter deficitario de sus inferencias lógicas, ellos podrían optar por defender la mayor racionalidad práctica ${ }^{50} \mathrm{de}$ los principios inducidos para identificar su sector ${ }^{51}$. Claro que, por las mismas razones, podría ocurrir lo contrario: que sean aplicables los principios de Sc y no Sa. Pero, con independencia de la (in)solvencia lógica de estas inferencias, ello ocurriría, nuevamente, por razones prácticas. Desde este punto de vista la pregunta acerca del conflicto entre dos reconstrucciones (la lógica centrada en lo intrasistemático) y la dogmática y judicial (centrada en lo intersistemático) podría ser respondida sosteniendo que aunque el conflicto no exista, algo de lo que tampoco estoy seguro, la reconstrucción lógica es insuficiente para dar cuenta de la práctica de los juristas. Ello así porque su foco principal ha estado puesto en lo intrasistemático y no en lo intersistemático y este foco ha impedido ver los límites que en la

\footnotetext{
${ }^{49}$ Inferencias que se relacionan con la derrotabilidad en sentido lógico.

${ }^{50}$ Sea como fuere que ésta se reconstruya.

${ }^{51}$ Esto significa que podría ser útil distinguir, como Cristina Redondo, entre derrotabilidad en sentido lógico y derrotabilidad en sentido práctico. Un principio lógicamente derrotable por otro (en este caso el que identifica a Sa por el que identifica a Sc) puede ser considerado por los juristas "inderrotable" pero desde un punto de vista práctico. Redondo, Cristina: "Reglas "Genuinas" y Positivismo Jurídico", Analisi e Diritto, 1998, pp. 243-276; también en "Lógica y Concepciones del Derecho", op.cit., pp. 46-48.
} 
práctica tiene esta reconstrucción lógica. Los juristas usan sus teorías para reconstruir las condiciones en virtud de las cuales las soluciones previstas para un sistema no pueden (o pueden) extrapolarse a otros. Esta es la idea del principio: los juristas desarrollan disciplinas que les otorguen una base idónea para perfeccionar conceptos y elaborar teorías que ofrezcan criterios para mostrar qué "combinaciones sistemáticas" de normas son admisibles. Es decir, referidas a mostrar criterios para construir sistemas que resuelvan casos. Estos criterios son variados y pueden presentarse bajo aspectos un tanto oscuros. Por ejemplo, para seguir una terminología que Vernengo toma de Husserl, sosteniendo la existencia de "ontologías regionales" relativas a cada sistema normativo ${ }^{52}$. Pero de esta oscuridad de la dogmática no se sigue que las ideas de autonomía y unidad expresen intuiciones que no valga la pena explorar. Después de todo, la exploración de estas intuiciones podría ser una manera de reconstruir las diversas imágenes que del conocimiento jurídico y su objeto los juristas presuponen en el uso de estas ideas.

${ }^{52}$ Vernengo, Roberto: "La Función Sistemática de la Norma Fundamental”, Revista Jurídica de la Facultad de Derecho de Bs As, Bs As, I-II, 1960, pp. 211;222-225. 
$\triangle \quad$ DOXA 25 (2002) 